\title{
Le rôle du formateur dans l'articulation des compétences acquises sur simulateur et des compétences cibles (" terrain »)
}

Le cas du moniteur dans la formation de pilotes militaires d'hélicoptères - armée de Terre

Trainer's role for linking competencies acquired through simulation and target competencies for the field. A case study in training helicopters military pilots

Janine Rogalski et Boris Colin

\section{(2) OpenEdition}

\section{Journals}

Édition électronique

URL : http://journals.openedition.org/activites/3333

DOI : 10.4000/activites.3333

ISSN : $1765-2723$

Éditeur

ARPACT - Association Recherches et Pratiques sur les ACTivités

Référence électronique

Janine Rogalski et Boris Colin, «Le rôle du formateur dans l'articulation des compétences acquises sur simulateur et des compétences cibles («terrain ») », Activités [En ligne], 15-2 | 2018, mis en ligne le 15 octobre 2018, consulté le 02 mai 2019. URL : http://journals.openedition.org/activites/3333 ; DOI : 10.4000/activites.3333

Ce document a été généré automatiquement le 2 mai 2019.

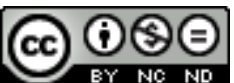

Activités est mis à disposition selon les termes de la licence Creative Commons Attribution - Pas d'Utilisation Commerciale - Pas de Modification 4.0 International. 


\section{Le rôle du formateur dans} l'articulation des compétences acquises sur simulateur et des compétences cibles (« terrain »)

Le cas du moniteur dans la formation de pilotes militaires d'hélicoptères - armée de Terre

Trainer's role for linking competencies acquired through simulation and target competencies for the field. A case study in training helicopters military pilots

Janine Rogalski et Boris Colin

\section{NOTE DE L'ÉDITEUR}

Article soumis le 8 février 2018, accepté le 28 juin 2018

\section{Introduction}

La question du transfert des acquisitions sur simulateur à l'activité en situation professionnelle réelle est une vieille question, posée dès la conception des premiers simulateurs, dans le domaine de l'aviation (Grau, Doireau, \& Poisson 1998, p. 362 pour le domaine militaire). Un bref rappel historique en a été fait par Leplat dans sa préface à (Pastré, 2005, pp.2-3). Précisons d'emblée que nous parlerons de terrain plutôt que de réel, car un simulateur est tout à fait réel, de même qu'une situation de simulation, appelant, ainsi que l'a souligné Savoyant (2005), une activité tout aussi réelle de l'élève et du formateur. Précisons dès à présent que formateur et élève travaillent dans un hélicoptère à doubles commandes, dans une relation qui sera retrouvée ultérieurement en pilotage à deux ${ }^{1}$. 
2 Le passage du simulateur au terrain a été essentiellement abordé dans la littérature du point de vue du transfert des apprentissages des élèves et très peu du point de vue des formateurs ${ }^{2}$. Pourtant de nombreux bilans convergent sur le fait que la manière dont le simulateur est utilisé est essentielle (McCauley, 2006, p. 31 ; Salas, Bowers, \& Rhodeniser, 1998). Cela comporte d'une part le choix et l'organisation des tâches proposées aux élèves, et, d'autre part, la manière dont le formateur gère la réalisation de ces tâches par l'élève.

3 Nous nous intéressons ici à la formation initiale au pilotage d'hélicoptère dans le cadre de l'armée de Terre (EALAT Dax) 3 . Cette formation a deux visées : la préparation au brevet de pilote professionnel civil et la constitution des prérequis de pilotage qui conditionnent la poursuite de la formation comme pilote militaire (avec un complément de formation à Dax, puis une «transformation » sur type d'appareil, système d'armes, sur une autre base - Le Luc).

4 La préparation au brevet de pilote professionnel fait dépendre la formation à l'EALAT de Dax des normes de l'aviation civile. Cela concerne, d'une part, l'empan des tâches visées et leur normalisation et, d'autre part, la fidélité du simulateur - en particulier en termes de fidélité des commandes et de qualité de l'information visuelle qui en dépend, même si le mouvement n'est pas représenté ${ }^{4}$. La formation articule séances sur simulateur et vols réels, visant à la maîtrise des manœuvres au cœur du pilotage. Il s'agit d'une formation très encadrée, du double point de vue des fortes normes de l'aviation civile (validation par la DGAC) et des attentes élevées vis-à-vis des élèves pilotes militaires (il faut relever qu'une forte sélection précède l'entrée en formation des élèves). Par ailleurs, l'activité d'un pilote militaire a une forte dimension collective : conduite en équipage à l'intérieur de l'hélicoptère et intégration des hélicoptères militaires dans un dispositif opérationnel complexe.

5 L'activité du formateur est elle-même encadrée au niveau de la logique de progression des tâches élèves et pour partie de leur gestion. Elle reste relativement ouverte quant à la mise en œuvre in situ des démonstrations d'exercices et du guidage de l'activité de l'élève.

Si du point de vue de l'élève, les heures sur simulateur sont décomptées comme heures de pilotage, il n'en reste pas moins que les conditions de son activité diffèrent (ne serait-ce que par la présence d'un risque concret, de perturbations possibles, et d'une autre variabilité des situations lors des vols réels). Le formateur doit lui aussi adapter son activité selon qu'il s'agit d'une gestion didactique en simulateur ou en vol réel, situations qui ne présentent ni les mêmes opportunités ni les mêmes contraintes. C'est cette adaptation entre simulateur et terrain qui est la problématique qui oriente la suite de l'analyse ${ }^{5}$.

7 La section 1 résume des acquis et questions issus de la littérature professionnelle et de la recherche, puis les cadres théoriques qui sont utilisés pour analyser l'activité du formateur. Des documents professionnels sur le pilotage d'hélicoptère et sur la formation des pilotes fournissent des données sur la prescription dans le domaine étudié, que ce soit dans l'établissement de standards pour les pilotes ou de consignes ou conseils sur la tâche du formateur. En revanche, les recherches sur la formation au pilotage d'hélicoptère s'avèrent rares: elles concernent les ressources et ne font qu'évoquer le rôle du formateur. Des travaux réalisés dans d'autres situations de formation à la conduite de mobiles peuvent éclairer la fonction du formateur dans le passage du simulateur au terrain. Deux cadres théoriques de psychologie ergonomique et de didactique 
professionnelle sont utilisés pour analyser l'activité du formateur en général. La psychologie ergonomique apporte des éclairages - et des questions - sur l'objet de la formation: elle permet en particulier de situer ce pilotage dans des catégories pour lesquelles existent des études antérieures (la gestion d'environnement dynamique et plus spécifiquement la conduite de mobiles). La didactique professionnelle est exploitée pour l'analyse de l'activité du formateur. En retour, l'étude du cas étudié dans la section suivante vise à contribuer aux recherches sur l'activité des formateurs dans des dispositifs de formation de professionnels.

8 La section 2 développe ensuite l'étude du rôle des moniteurs (selon la terminologie de l'EALAT) dans l'articulation de la formation des élèves sur simulateur et sur « le terrain ». Le second auteur, pilote et formateur, a répondu à un ensemble de questions du premier auteur, orientées par les cadres théoriques et par les éléments de la littérature. La section 2 présente une organisation de ses réponses. Les déterminants de l'activité des moniteurs y sont précisés. Du côté de la situation (déterminants extrinsèques), il s'agit des élèves dont ils ont la charge, des prescriptions sur l'organisation des procédures et manœuvres que les élèves doivent successivement maîtriser, de l'encadrement préconisé ou organisé de la gestion des séances. Du côté des moniteurs (déterminants intrinsèques), il s'agit en particulier de la place de leurs propres compétences de pilotage, de leur connaissance de l'activité sur simulateur par rapport au pilotage de l'hélicoptère réel, et de leurs connaissances sur les processus de formation / apprentissage. L'exploitation qu'ils peuvent faire des outils de guidage de leur activité est un déterminant au regard d'une visée de standardisation de la formation. On évalue aussi l'espace d'autonomie de leur activité, dans l'étayage puis dans l'évaluation de l'activité des élèves dont ils ont la charge, et l'effacement de l'étayage jusqu'à leur autonomie.

La discussion, dans la section 3 qui suit, porte sur le domaine de pertinence des analyses proposées de l'activité de moniteur dans le cas du pilotage d'hélicoptère, pour en inférer des éléments sur les apprentissages dans d'autres types de situations professionnelles. Il s'agit plus particulièrement des situations dynamiques qui sont marquées par une grande place des activités perceptivo-motrices; c'est-à-dire des situations dans lesquelles existe un composant important de contrôle manuel direct. Le formateur doit agir pour que l'élève à la fois arrive à automatiser des procédures et reste adaptable pour répondre à l'ouverture de l'environnement dynamique (variabilité des paramètres de situations, possibles perturbations externes, dysfonctionnements éventuels). L'élève doit se construire des schèmes d'action - au sens de Vergnaud après Piaget - c'est-à-dire des " invariants de l'organisation de l'action dans une classe de situations", même si l'action peut différer dans ses modalités d'exécution (Rogalski, 2011).

10 Au-delà de l'étude de cas, de nombreuses questions sont ouvertes du point de vue de la recherche sur l'activité des formateurs. Ainsi, la dimension métacognitive (Valot, Grau, \& Amalberti, 1993) dans la formation mérite d'être analysée de manière approfondie. Cela concerne en particulier l'exploitation didactique de la comparaison entre l'autoévaluation des élèves et celle effectuée par les moniteurs : un indicateur d'apprentissage peut être le processus de la convergence de ces évaluations au cours de la formation. Cela concerne aussi l'intervention didactique sur d'autres activités réflexives, portant moins sur la technique que sur les autres niveaux de l'activité de pilotage. Le double concept d'étayage et d'effacement peut être travaillé pour préciser davantage la méthodologie générale qui est suivie dans la formation étudiée, sous le schéma «démonstration / exécution». Une visée pourrait être de contribuer à la conception continuée d'aides à 
l'activité des formateurs, à travers les ressources que constituent les guides de l'instructeur. En amont de l'activité des formateurs, c'est un retour sur leur propre formation qui peut être visé, en particulier à travers des outils pour analyser leur propre activité, leur permettant d'exploiter leur espace de liberté pédagogique et didactique.

\section{Données de la littérature et cadres d'analyse de l'activité du formateur}

\subsection{Connaissances acquises sur le pilotage d'hélicoptère, la formation et le rôle de la simulation} considéré comme une tâche particulièrement difficile. Étudiant la place des circuits de prise d'information visuelle dans la navigation, Sullivan, Yang, Day et Kennedy (2011, p. 871) reprennent ainsi l'évaluation d'une de leurs références (Padfield, 1992, p. 116) sur la formation: "dans le domaine de l'aviation, apprendre à piloter un hélicoptère est généralement considéré comme la tâche la plus exigeante et la plus difficile » (notre traduction [NT]). ${ }^{6}$ La même évaluation est présente dans un document de l'aviation civile (Transport Canada, 2006), qui précise que pour les néophytes, « une des manœuvres les plus difficiles à maîtriser est le vol stationnaire - garder une position stable 3 à 5 pieds au-dessus du sol ».

Une raison citée est que l'hélicoptère est par essence instable. La différence avec le pilotage d'aéronefs à voilure fixe est précisée dans une étude de la NASA sur les accidents d'hélicoptères (rotorcrafts) : «à la différence des pilotes d'aéronefs à voilure fixe, le pilote d'hélicoptère doit contrôler en permanence les quatre axes primairess ${ }^{7}$ qui sont fréquemment couplés [...] Le pilote doit donc utiliser à la fois ses deux pieds et ses deux mains $»^{8}$ (Harris, Kasper, \& Iseler, 2000, p. 29, NT). Pour le vol stationnaire (hover), l'apprentissage de la dynamique du système est nécessaire à l'atteinte de la stabilisation, qui suppose l'utilisation d'ajustements proactifs.

Pourtant, comparativement à ce qui s'est passé pour la place de la simulation dans la formation au pilotage d'avion, les recherches concernant les hélicoptères restent rares, comme l'indiquent plusieurs articles de synthèse. En 1992, Hays, Jacobs, Prince et Salas n'identifiaient que 7 recherches portant sur les hélicoptères à partir de 247 articles, rapports de recherche et rapports techniques sur l'utilisation de la simulation dans le champ de l'aviation (Hays, Jacobs, Prince, \& Salas, 1992). Ces recherches semblaient ne pas contredire les conclusions portant sur les avions: utilité de la simulation pour l'apprentissage, effectivité d'une formation conduite au rythme des acquisitions ( performance-paced) plus que d'un programme fixé a priori (lock-step).

14 Stewart II, Dohme et Nullmeyer (2002) présentent une revue de la littérature sur le transfert d'apprentissage du simulateur au vol réel pour la formation initiale (ab initio training). Ils soulignent la difficulté de déterminer ce qui contribue à un transfert positif entre les «tâches procédurales » et les «tâches de vol » (procedural and flying tasks), qui sont d'ailleurs en interaction. Ils reprennent la conclusion de Salas, Bowers et Rhodeniser (1998) sur la stagnation des recherches dans le domaine :

« des recherches plus récentes [...] ont été l'exception et non la règle. Ceci est particulièrement le cas pour la recherche sur la formation des équipages des aéronefs à voilure tournante » ${ }^{9}(\mathrm{NT})$. 

mouvement pour les plateformes de simulation et la qualité de la visualisation en relation avec les processus de balayage visuel (visual scan).

Un bilan de McCauley (2006), dans le contexte de l'armée américaine, relève que le mouvement pour les simulateurs est une aide pour piloter le simulateur lui-même (particulièrement pour les pilotes expérimentés, et dans le cas de perturbations externes), mais qu'il n'y a pas d'évidence d'un transfert d'apprentissage du simulateur au vol réel. Cela rejoint des conclusions de la revue de Hays et al. (1992, op. cit.). Les auteurs y soulignent qu'une leçon importante que l'aviateur doit apprendre est d'ignorer ou de supprimer les informations (input) vestibulaires et de se fier à ses instruments, tout en se demandant s'il est besoin de simuler le mouvement pour apprendre à l'ignorer? Ce rapport ajoute qu'il n'y a pas non plus de données fiables sur le fait qu'une base de simulation avec mouvement préviendrait la forme de cinétose qu'est « le mal du simu ».

17 La fidélité visuelle du système de simulation est, quant à elle, toujours considérée comme essentielle dans le cas des simulateurs pour l'apprentissage dans le domaine de l'aviation et particulièrement du pilotage d'hélicoptère. La figure 1 propose un exemple de balayage visuel.

Les schémas de balayage visuel, adaptés au moment de vol, sont évidemment identiques en simulateur et en hélicoptère. Il en est de même pour la nécessité de fidélité des effets des commandes. Ce sont deux conditions cruciales de la pertinence des situations d'apprentissage des manœuvres de base et de la navigation ${ }^{10}$. Proctor, Bauer et Lucario (2007), questionnant les conditions de la formation au pilotage d'hélicoptère à partir de serious gaming, parlent plus largement de fidélité du terrain, en particulier pour les appréciations de distance et de vitesse. Relevons leur conclusion :

« en matière d'épilogue, la Bristow Academy a adopté depuis un simulateur de vol Bell 206 comme composant de son programme de formation, et aucune des trois approches de serious games présentées plus haut $\left.{ }^{(\mathrm{NT}}\right)^{11}$. 
Figure 1 : Un schème de balayage visuel, d'après le manuel de l'aviation civile du Canada. Figure 1: Schema of visual scan, from a manual of the Canadian civil aviation

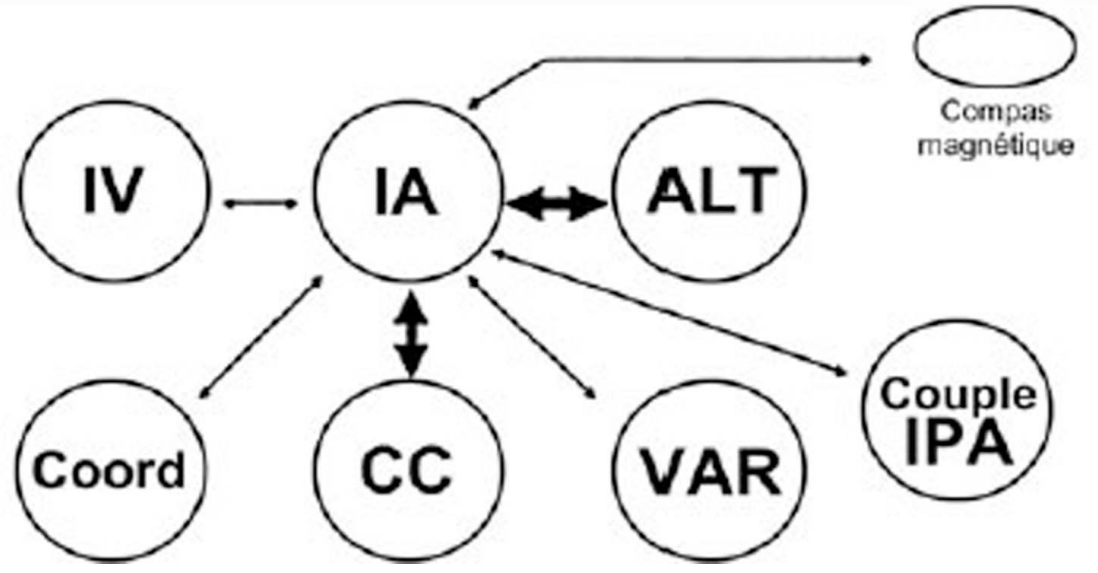

Balayage pour le vol rectiligne en palier.

Le balayage répond à trois questions :

- De quels renseignements j'ai besoin pour le vol rectiligne en palier ;

- Quels sont les instruments qui me donnent ces renseignements ;

- Les renseignements sont-ils fiables?

Les flèches en gras montrent la modalité de balayage visuel que le pilote utilise le plus fréquemment pour obtenir l'information voulue.

Les flèches en traits plus légers indiquent un balayage visuel moins fréquemment répété des instruments d'appoint.

Toutefois des auteurs comme Yang, Kennedy, Sullivan et Fricker (2013) considèrent que, dans les formations qu'ils ont analysées, la stratégie de balayage visuel ne reçoit pas la priorité qu'elle devrait avoir et qu'on aide peu les formateurs à faire un retour efficace à l'élève sur ce point ${ }^{12}$. Ces auteurs proposent d'identifier quelle information leur permettrait de repérer en temps réel sur quoi l'élève porte son attention visuelle, et d'évaluer plus aisément ce qu'ils appellent la "flexibilité attentionnelle » (attentional flexibility) à la suite de Bellenkens, Wickens et Kramer (1997). Ils relèvent le besoin de concevoir des outils pour aider les formateurs à faire des retours plus efficaces à l'élève sur ce point, et pour leur permettre de s'appuyer pour la formation à la navigation sur une caractérisation des patterns de balayage visuel entre l'extérieur et l'intérieur du cockpit (visual scan) (Sullivan, et al. 2011, repris par Yang et al., op. cit.) ${ }^{13}$.

En fait, de manière générale, les publications de recherche soulignent le rôle clé que joue, d'une part, l'organisation des situations de formation (instructional design) et, d'autre part, la qualité de la mise en œuvre par les formateurs (par ex.: McCauley, 2006; Salas et al., 1998 ; Samurçay \& Rogalski, 1998). Elles rendent aussi visible en creux que l'activité des formateurs est la Cendrillon des recherches du domaine.

21 En revanche, la littérature professionnelle publiquement accessible propose deux types de ressources pour la formation au pilotage d'hélicoptère : l'une est orientée vers les candidats pilotes et l'autre vers les formateurs. Ces documents professionnels proposent aux formateurs - après qu'ils aient été en formation - des guides relativement voire très détaillés pour la conduite des situations didactiques. Ces guides sont centrés sur les tâches à enseigner (côté formateur)/ apprendre (côté élève) et se déclinent essentiellement pour des conditions de vol réel. La philosophie sous-jacente étant qu'on apprend la même chose de la même manière dans les deux types de situations simulateur ou vol réel - ou en tout cas qu'il s'agit de la même visée et des mêmes ressorts 
didactiques. Les deux dimensions, au sens utilisé par Lépinard (2014), sont présentes : la dimension pédagogique propose un modèle de démonstration par l'instructeur / exécution par l'élève, et insiste sur l'établissement d'une bonne relation de coinvestissement dans la formation; la dimension didactique s'exprime par un contenu détaillé du déroulement des différents exercices ou phases de l'apprentissage, en rappelant le critère prioritaire de sécurité - effectif en cas de vol réel ; il n'y a pas de commentaire particulier pour la transposition de ce critère (du point de vue de l'élève) en situation de simulation. De manière dominante, ces documents sont centrés sur ce qui est à acquérir comme pilote - non sur le rôle spécifique de la simulation dans la réalisation de cette visée.

En fait, la place de la simulation dans l'apprentissage du pilotage d'hélicoptère peut être directement située par rapport à deux contextes : le contexte de l'aviation et un contexte plus général de conduite de mobiles dans lesquels le pilote est physiquement présent. Les deux domaines relèvent par ailleurs d'une catégorie générale de gestion d'environnements dynamiques, pour laquelle existe un très grand corpus de recherches, dans une diversité de domaines. Il est alors possible de s'appuyer sur des modèles pour l'analyse de l'activité prolongeant le modèle de double échelle de Rasmussen (Chauvin \& Rogalski, 2011), pour proposer un modèle du formateur comme gérant lui-même un environnement dynamique (ici : l'interaction entre l'élève et l'hélicoptère).

Nous allons présenter les modèles génériques que nous utilisons comme appui des analyses particulières du rôle du formateur entre simulateur et terrain dans la formation au pilotage d'hélicoptère $a b$ initio. Ils s'inscrivent dans la lignée de didactique professionnelle de Pastré (Pastré, 2011 ; Pastré et al., 2006).

\subsection{Le modèle de double régulation de l'activité en situation de formation}

Le modèle de double régulation de l'activité, introduit et développé par Leplat (1997) et spécifié par Rogalski (2003) pour le cas de l'enseignement et la formation, distingue-les (co)-déterminants de l'activité qui relèvent du sujet (dimension « intrinsèque ») de ceux de sa situation de travail (dimension "extrinsèque »). Ces déterminants produisent des effets dans ces deux dimensions: sujet et situation. Ces effets modifient en retour les conditions de l'activité en cours. Dans une situation de formation professionnelle, deux acteurs (au moins) sont impliqués : l'élève et le formateur. On peut utiliser le même cadre de la double régulation pour questionner les activités de l'un et de l'autre, activités qui sont fortement articulées.

\subsubsection{Des déterminants communs dans l'activité de l'élève et celle du formateur}

Le dispositif de formation en est un déterminant commun : l'activité de l'élève comme celle du formateur doit viser l'acquisition des compétences attendues vis-à-vis des tâches qui sont la cible de la formation. Il propose ou impose aussi des ressources et des contraintes, en particulier le temps global de formation et les moyens disponibles - dont font partie, pour ce qui concerne le pilotage, les heures de vol et les simulateurs.

La visée de l'élève est (ou devrait être) d'aboutir à ce que ses compétences rejoignent celles attendues. C'est donc la dimension constructive de son activité qui est centrale (Samurçay \& Rabardel, 2004, pp.166-167). La construction des compétences est 
accompagnée d'une formation théorique. Dans la composante pratique de la formation, elle se développe via l'action en situation réelle, ou transposée de la situation-cible. Il y a alors également une visée de production : l'hélicoptère doit être piloté. L'intervention du moniteur sur ce qui fait problème est alors à la fois source des acquisitions pour l'élève et moyen de les évaluer, pour le formateur.

Le formateur partage la même visée générale. Toutefois l'objet de son action didactique diffère : pour lui, il s'agit de l'activité même de l'élève en tant que source d'apprentissage pour ce dernier. Il a deux moyens d'action principaux: les tâches qu'il donne et l'intervention didactique sur l'activité de l'élève dans la réalisation de ces tâches. Un autre moyen d'action de formation, qui peut selon les situations être plus indirect, mais qui reste central, est la personne même du formateur, à travers ses actes et leur explicitation.

Pour les deux acteurs, élève et formateur, on peut considérer le simulateur comme un instrument pour le processus formation / apprentissage. Dans le contexte de notre étude, le simulateur est pour l'élève un moyen d'apprendre à piloter un hélicoptère réel, avec un but à plus long terme: l'intégration dans des missions militaires. Pour le moniteur, le simulateur est un outil qui, à la fois, lui offre un potentiel didactique particulier pour former de «bons » schèmes de pilotage chez l'élève et lui permet de se centrer sur cette visée, dégagé d'assurer la sécurité du vol avec surveillance de la radio (tâche présente en formation sur le terrain), même si l'on intègre cette dimension dès le début avec l'élève avec l'annonce des sécurités à assurer. Mais le moniteur s'interdit en simulation toute forme de crash qui pourrait être banalisé (negative training) - et sur le plan pédagogique perçu comme un échec.

\subsubsection{L'articulation de l'activité de l'élève et du formateur}

Un élément central dans l'articulation de l'activité de l'élève et du formateur est la convergence des buts que nous venons de souligner. Cette convergence n'est pas donnée, elle est à constituer dans une forme d'alliance entre ces acteurs, à laquelle le formateur doit être attentif. Un des retours de l'activité de ce dernier concerne ainsi la cohérence des buts et l'évitement des malentendus didactiques. Cela touche à la fois la dimension pédagogique des relations élève / formateur et la dimension didactique de gestion des tâches / activités des élèves - en reprenant une distinction de Lépinard entre ces deux dimensions (2014, p. 51 sq.). Les briefings et les débriefings sont des moments privilégiés pour cette mise en cohérence des buts de chacun. La convergence des buts peut être plus problématique dans l'exploitation des situations de simulation, et il peut y avoir besoin d'actions spécifiques pour la constituer. En particulier, il faut éviter que pour l'élève le but soit d'apprendre à contrôler la situation de simulation (à l'instar des logiciels de jeux) alors qu'il s'agit d'apprendre à agir dans la situation de référence.

Dans l'activité du formateur, se pose ainsi le problème de la posture de l'élève, c'est-à-dire son rapport à la situation de simulation. Le formateur a en charge de provoquer ou contrôler un enrôlement de l'élève en ce sens. Des propriétés de l'élève (« qualités » dans Rogalski, 2011) peuvent favoriser ou entraver cet enrôlement. L'élève déjà professionnel (formation à l'emploi, spécialisation, qualification de type...) importe son expérience dans la situation de simulation et situe ce qu'il y apprend dans son activité de terrain future (situation de référence, ou situation cible); a contrario il peut être plus critique sur les situations de simulation. En formation ab initio, c'est l'organisation de la situation de 
simulation qui contribue à un tel enrôlement: le simulateur (et ses propriétés) est un composant et non le tout de la situation de simulation, comme l'ont développé Samurçay et Rogalski (1998). Les caractéristiques personnelles du formateur sont aussi un composant de l'enrôlement, en particulier ses compétences de pilotage interviennent dans sa légitimité perçue.

\subsection{La formation comme gestion d'un environnement dynamique " ouvert »}

31 Un modèle de l'activité de formation comme gestion d'un environnement dynamique (GED) ouvert (Rogalski, 2003, pour le cas de l'enseignant ; Vidal-Gomel \& Rogalski, 2009, pour le formateur en conduite automobile), permet de bénéficier des outils d'analyse déjà élaborés. En fait, pour agir, le formateur a besoin de deux modèles : un modèle de l'objet de l'action de l'élève - ici le pilotage d'hélicoptère -, et un modèle de l'intervention didactique, qui s'appuie lui-même sur un modèle des compétences attendues et de leurs apprentissages. Pour analyser des situations de formation, la didactique professionnelle exige ainsi de s'appuyer sur l'expertise dans le domaine professionnel concerné (professionnels experts, formateurs, documentation professionnelle, acquis des recherches dans le domaine). Cette analyse concerne la conception des tâches et la dynamique des séances de formation.

$\mathrm{Du}$ point de vue de la conception, quels sont les procédés didactiques en jeu dans l'organisation de ces tâches, en particulier dans l'ordonnancement selon leur complexité? Quels sont les processus de transposition en jeu dans le passage des situations-cibles de l'apprentissage aux situations de formation (découpage d'unités, découplage d'interactions, focalisation sur un composant de la tâche-cible, cf. Samurçay \& Rogalski, 1998). Quel est le processus inverse de transfert des acquis des situations de formation au réel professionnel? Cette dernière question ne se pose pas seulement pour les situations de simulation, elle concerne tout dispositif institutionnel de formation ; elle ne porte pas que sur des éléments techniques, mais aussi sur la posture par rapport aux erreurs, à la prise de risque et par rapport à la dimension toujours collective de l'activité.

Du point de vue de la dynamique d'une séance de formation - en aval de la conception des situations didactiques-, une question particulière interroge les relations entre simulateur et terrain. Un modèle de régulation de l'action de l'élève constitue une entrée dans l'analyse de l'intervention du formateur. 


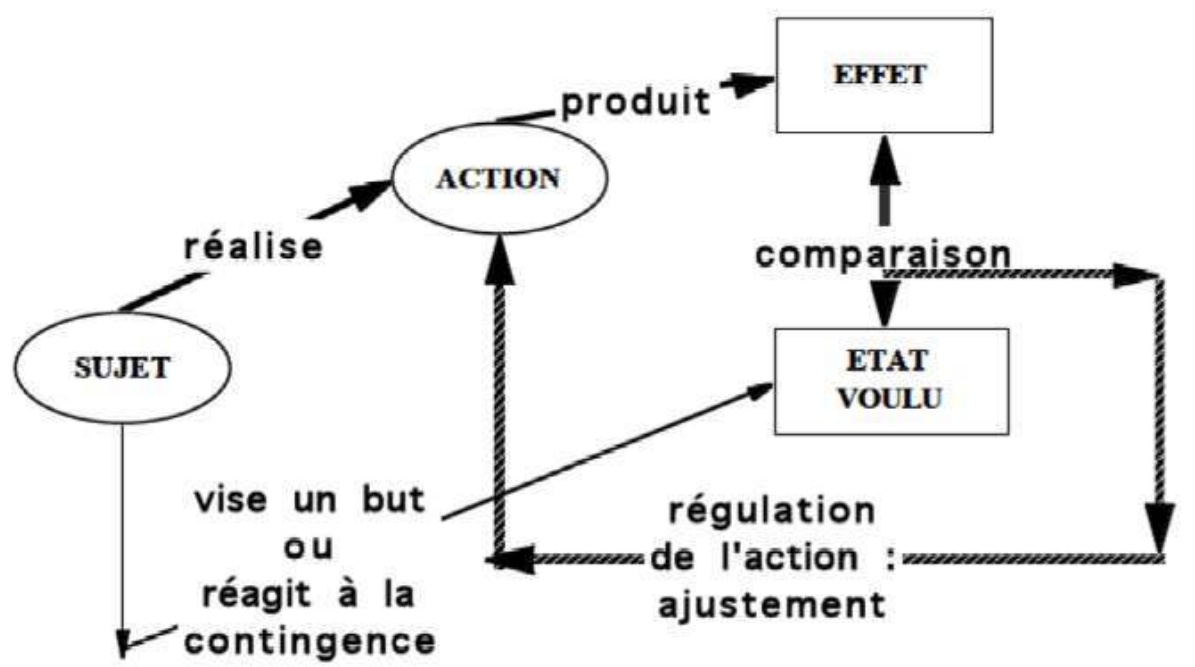

Présenté dans la figure1, ce modèle vient directement de la conception constructiviste de Piaget, reprise en didactique professionnelle (Pastré et al., 2006). Il concerne l'action particulière en cours; elle peut - si c'est utile - être analysée en une organisation d'opérations de niveau inférieur (les balayages visuels par exemple, ou l'utilisation de commandes).

Il s'agit d'un modèle de régulation dans une temporalité relativement courte. L'acteur (sujet) a un but à atteindre (qu'il s'est donné ou qu'on lui a donné), il réalise une action destinée à atteindre ce but (état voulu c'est-à-dire état-cible du point de vue du sujet). Il compare l'effet de son action à cette cible, et en fonction de la comparaison ajuste son action. La boucle s'arrête quand l'état atteint est l'état-cible ou un état "suffisamment " proche. Ce processus de régulation peut aussi représenter la réaction à la contingence : l'action peut être déclenchée par une perturbation externe. Si seul l'élève est acteur, ce modèle de régulation locale peut représenter un apprentissage par l'action (comme dans le cas de l'usage autonome d'un simulateur).

La didactique professionnelle a introduit la fonction de médiation remplie par le formateur, en transposant dans le cas des acquisitions professionnelles l'approche théorique de Vygotsky du développement vers des savoirs au sens large. Ce développement a lieu si les problèmes posés au sujet en formation sont assez proches de ce qu'il fait / peut déjà faire, mais pas trop pour qu'il puisse y avoir quelque chose à apprendre. En termes vygotskien: le problème doit se situer dans la Zone Proximale de Développement (ZDP) de l'élève (cf. Vygotski, 1997, chap. 6).

On peut utiliser le schéma précédent pour distinguer a priori des moments d'intervention du formateur : donnée ou repérage du problème (alerte); identification du but; aide à l'exécution de l'action; contrôle des effets, en tenant compte du fait qu'ils peuvent être " primaires» (visés) ou « secondaires » (des effets de bord de l'action - ou side effects); orientation de l'ajustement de l'action et/ou aide à sa réalisation - cela concerne la temporalité de l'action, cruciale dans les environnements dynamiques à tempo rapide, ainsi que son organisation en manœuvres (Boccara, Vidal-Gomel, Rogalski, \& Delhomme, 2015, p. 22). 

notion d'étayage (scaffolding) proposée par Bruner (2002): il s'agit d'une analyse de l'activité du formateur, mieux sachant ou mieux capable que l'élève, pour permettre à ce dernier de réaliser avec aide ce qu'il ne pourrait pas réaliser seul. La notion d'étayage a été élaborée - initialement sous le nom de tutoring - dans le contexte du développement de l'enfant (Wood Bruner \& Ross, 1976; Bruner, 2002) et étendue à l'éducation à tous les niveaux. Elle a été transposée pour l'étude de l'activité de formateurs dans le contexte para-professionnel de la conduite automobile (Vidal-Gomel, Boccara, Rogalski, \& Delhomme, 2008).

Parmi les six types d'intervention proposés par Bruner, trois types concernent directement la réalisation des tâches ${ }^{14}$ :

1. la réduction des degrés de liberté ;

2. la signalisation des caractéristiques déterminantes de la tâche - de l'orientation au contrôle de l'action ;

3. la démonstration (le formateur montre ce qui doit être fait).

Il y a apprentissage si l'étayage s'efface progressivement (fading) jusqu'à l'action autonome de l'élève. Cet effacement est dépendant des tâches: l'intervention sur l'ajustement de l'action se poursuit plus longtemps pour les tâches les plus délicates (Boccara et al., 2015, op. cit.). Nous verrons qu'il faut ajouter un type d'intervention: le modelage, dans lequel le formateur guide physiquement l'action au plus près de la réalisation sensori-motrice.

\section{Le rôle des moniteurs de pilotage d'hélicoptère dans le contexte de la formation $a b$ initio dans l'armée de Terre}

Le cadre général de la situation de pilotage est d'abord présenté avant d'aborder la thématique des relations entre simulation et vol réel. Les déterminants du côté sujets (moniteurs) et du côté situation (simu ou terrain) sont ensuite détaillés ; en particulier les prescriptions concernant les tâches (exercices / séances) et les guides pour l'activité des moniteurs sont analysés comme des outils pédagogiques et didactiques. La gestion par les moniteurs des relations entre formation sur simulateur et formation en vol réel est l'objet de la seconde partie de cette section.

\subsection{Cadre général, déterminants et outils pour l'activité des moniteurs}

41 Il s'agit d'une formation qui a deux objectifs : l'obtention par les élèves d'une licence professionnelle civile (CPL - commercial pilot license) et la délivrance d'un brevet militaire.

L'objectif civil introduit des contraintes générales de l'aviation civile nationale (DGAC, 2001), européenne et internationale. Ainsi tout simulateur de vol pour la formation initiale est validé - et régulièrement contrôlé - par la DGAC. D'autre part, seuls des instructeurs ayant une qualification de vol civile peuvent instruire les élèves (FIH - Flight Instructor Helicopter), que ce soit en vol réel ou sur simulateur. La formation de ces 
instructeurs - des moniteurs selon la dénomination de l'École - est assurée par un stage interne, lui-même validé par la DGAC.

L'objectif militaire vise la formation des élèves qui suivront ensuite une formation spécialisée, orientée vers la réalisation des différents types de missions militaires dans lesquelles les hélicoptères seront engagés comme moyens. On peut dire que la formation initiale aux manœuvres de pilotage, de circulation et de navigation doit faire en sorte que l'hélicoptère devienne un instrument pour réaliser les missions proprement dites. On y adjoint du vol sous SIL/JVN (Système d'intensification de lumière / jumelles de vision nocturne). Dans ces missions, la dimension collective est très importante : le plus souvent, les pilotes sont deux dans le cockpit et ils sont amenés à intervenir dans le cadre d'une patrouille (il s'y ajoute évidemment l'intégration dans un dispositif d'actions potentiellement bien plus large). D'où un besoin de standardisation dans la réalisation des tâches, pour la constitution d'anticipations appropriées de l'action d'autrui.

Dans le contexte du cas étudié, les moniteurs sont des pilotes militaires opérationnels, exceptionnellement réservistes. Ils interviennent aussi bien en régiment (pour les contrôles, les requalifications) qu'en École pour des types de vol spécifiquement militaires (vol tactique, combat, vol aux instruments en circulation aérienne militaire). Vis-à-vis des élèves, il s'agit donc de pilotes confirmés; on peut faire l'hypothèse raisonnable que ce statut contribue à leur impact sur les élèves, quel que soit le médium de la formation au pilotage: simulateur ou réel; on peut aussi penser que cette qualité permet des interventions métacognitives au-delà de celles - rares - qui sont explicitées dans les guides.

Les moniteurs sont par ailleurs formés à l'utilisation du simulateur, non pas que son pilotage diffère du vol réel, mais pour appréhender certaines nuances qui pourraient être perturbatrices, comme des différences entre le «vrai » et le «faux» sur des fonctions connexes au pilotage (ex.: un bouton d'éclairage qui n'a pas tout à fait les mêmes fonctions), ou comme des limites dans la modélisation des pannes, qui n'ont éventuellement dans le simulateur utilisé qu'une seule issue ${ }^{15}$ : le moniteur doit donc la connaître pour ne pas se lancer dans une démonstration qu'il ne pourrait conduire à son terme et qui serait ainsi vouée à l'échec. De plus, dans les démonstrations et l'étayage de l'activité, le moniteur doit travailler pour l'élève: il doit donc prendre en compte la parallaxe liée au visuel, car il aura alors une vision légèrement différente de celle de l'élève.

Les conditions DGAC conduisent à un contenu de formation encadré en termes des manœuvres ${ }^{16}$ que les élèves doivent être capables d'exécuter efficacement et en sécurité. Les scénarios (didactiques) restent libres, c'est-à-dire à la discrétion de l'École, qui a édité un guide de l'instructeur (le MANFORM, manuel de formation). L'orientation didactique relève d'une logique de progression, compatible avec le principe vygotskien d'une intervention du moniteur dans la zone proximale de développement de l'élève.

47 Tout au long de la progression, la formation de l'élève présente une structure permanente: éléments théoriques en jeu; acquis du travail précédent, étude d'une nouvelle manœuvre à partir de la démonstration par le moniteur. Dans la formation $a b$ initio il est précisé que pendant la démonstration l'activité de l'élève est: "écouter regarder - suivre aux commandes » les actions du moniteur, que ce dernier commente au fur et à mesure. Le moniteur fait ensuite exécuter la manœuvre par l'élève en le guidant pour aboutir à une réalisation réussie : il attire l'attention sur les éléments pertinents de la situation par rapport à la tâche à réaliser, prend éventuellement en charge une partie 
de la prise d'information et/ou des commandes. Il fait enfin faire une exécution autonome pour une évaluation de l'acquis, avec des droits à l'erreur limités.

Cette organisation régulière des séances peut être interprétée dans le cadre de la médiation didactique chez Bruner, bien que la référence n'y soit pas explicitement faite. En particulier, l'étayage au cours d'une exécution guidée ne doit être ni excessif ni insuffisant, pour rester dans la zone proximale de développement de l'élève ; le moniteur doit tirer l'élève de ce qu'il sait faire vers ce qu'il n'a pas encore acquis; l'étayage doit ensuite s'effacer (fading) en allant vers une autonomie de l'élève (suffisamment rapide, du fait d'une exigence d'efficience de la formation).

Enfin, les élèves constituent une autre dimension des déterminants de l'activité des moniteurs: ils ont été fortement sélectionnés, du point de vue des dimensions psychotechniques de l'action (entre autres dissociation de l'attention et coordination motrice) et du point de vue de la stabilité émotionnelle et de la motivation. Il s'agit a priori d'un élément important dans leur enrôlement dans la formation au pilotage, que ce soit en vol réel ou sur simulateur, et dans leur activité constructive par leur participation à leurs acquisitions théoriques et pratiques au cours des séances et hors des séances.

\subsection{L'activité encadrante du moniteur et l'instrumentalisation du simulateur}

\subsubsection{Enrôler}

50 L'utilisation du simulateur comme instrument de formation pour le moniteur et instrument d'apprentissage pour l'élève s'appuie sur une mise dans l'ambiance qui se veut la plus proche du vol réel: ils travaillent habillés de la même manière, équipés du casque de vol, du couteau coupe sangle, des gants, etc. Dans son guidage et son évaluation de l'élève, le moniteur se comporte comme également en vol réel (même s'il utilise des outils propres au simulateur en cours de séance). Du fait qu'il est noté comme en vol réel, l'élève a tout intérêt à avoir une attitude en simulation compatible avec l'apprentissage (une orientation constructive de son activité sur simulateur visant sa compétence au pilotage d'hélicoptère - et non à celui du simulateur qu'il considèrerait comme un jeu), au risque sinon de s'exposer à une mauvaise progression et au pire à sortir du stage. Les deux dimensions pédagogique et didactique de l'action du formateur sont ici en jeu, en forte interaction.

\subsubsection{Faire acquérir un « modèle des variables de commandes »}

51 L'intervention didactique technique commence par la contribution à la compréhension des notions dynamiques propres à l'hélicoptère. En effet, la dynamique d'un hélicoptère ne peut pas être comprise par analogie avec d'autres mobiles qui seraient plus familiers encore moins à partir de schèmes très primitifs (liés à des affordances) comme l'est celui du mouvement du volant pour faire tourner une voiture. Les notions fondamentales doivent donc être enseignées, car elles sont essentielles à l'apprentissage du pilotage. Elles sont apprises d'abord en théorie, en cours collectifs présentiels. Puis, dès le premier vol - qui se fait sur simulateur -, elles seront montrées. La mécanique du vol hélicoptère veut qu'une action sur une commande apporte un effet primaire (recherché) et un effet secondaire (à compenser par une autre commande). Le moniteur décortique donc chaque 
effet de commande, fait constater l'effet primaire et l'effet secondaire, puis va faire faire l'exercice à l'élève.

La correction de l'effet secondaire est dans un premier temps à la charge du moniteur puis très rapidement l'élève intègre la correction à apporter. On voit ici jouer une fonction de " découplage » : l'élève n'a d'abord à sa charge qu'un composant de l'action, un autre étant assuré par le formateur (Samurçay \& Rogalski, 1998). On peut penser que l'effacement de l'étayage peut être relativement plus rapide du fait de la sélection initiale des élèves.

Dès le premier travail moniteur / élève, il y a construction chez ce dernier d'un modèle interne des variables sur lesquelles agissent les commandes (roulis, tangage, lacet) et de l'attitude de l'hélicoptère, le conduisant à pouvoir simuler mentalement les effets d'actions sur les commandes. Ce modèle est donc mis en place par le moniteur dès le premier briefing, montré en vol simulé puis en vol réel. L'élève peut ainsi reproduire lors de son travail personnel (le soir, en chambre) les gestes qu'il a appris en journée. On peut appeler cela du " chair flying ». C'est un élément essentiel pour une progression régulière ${ }^{17}$ . La répétition au sol permet chez l'élève l'instauration d'un réflexe : en vol, il aura alors plus de ressources disponibles pour travailler d'autres éléments du pilotage. Cet apprentissage mental contribue à la constitution d'un schème d'utilisation du système des commandes et des informations visuelles, de manière proactive (en anticipation) : il est donc important que le moniteur détecte toute déviance pour éviter l'apprentissage de mauvais mécanismes ou réflexes, qui seraient des schèmes dangereux pour la suite. On peut faire l'hypothèse que l'échec d'un petit pourcentage d'élèves au début de leur formation au pilotage s'expliquerait par l'absence de constitution d'un tel modèle interne, par manque de compréhension suffisante de la dynamique et/ou par insuffisance ou manque de pratique mentale.

\subsubsection{Lier compréhension et pilotage}

Le lien entre la compréhension et le pilotage est une constante dans la formation. Par exemple ce lien est souligné dans les documents professionnels sur formation au pilotage d'hélicoptère dans l'armée US, à propos d'une situation complexe, celle du risque de rollover (un tonneau pour hélicoptère... ${ }^{18}$ ) : «avant d'effectuer des opérations dans un environnement de type boue / tourbière / toundra, il est important de comprendre les caractéristiques du rollover dynamique " (NT) ${ }^{19}$. En fait, chaque situation de pilotage nécessite une compréhension des problèmes que l'on peut rencontrer, qu'il s'agisse de les traiter (via le simulateur) ou de les éviter (quand ils ne sont pas simulables).

Le moniteur peut se servir du simulateur comme outil didactique pour aider l'élève à la fois à la compréhension des éléments en jeu, à leur identification dans la situation et à un pilotage sûr et efficace. Par exemple, lors d'un décollage vertical proche d'obstacles, l'élève doit s'assurer de la bonne verticalité de la trajectoire en prenant de bons repères. Le moniteur peut utiliser la fonction «rejeu de trajectoire " pour montrer à l'élève la trajectoire qu'il a réellement effectuée. Si on se réfère au schéma d'ajustement de l'action (Figure 2), il s'agit d'une intervention de guidage en donnant à l'élève le moyen de distinguer l'action voulue (la « bonne trajectoire » qu'il cherchait à faire et celle réalisée), ce feedback étant un moyen très important de l'ajustement de l'action en se servant de repères initialement peu ou mal utilisés. Le simulateur peut aussi être utilisé pour dédramatiser (quand nécessaire) la proximité d'obstacles. Le simple rejeu de trajectoire 
est ainsi utilisé pour lever les doutes de l'élève quant à la distance entre l'appareil et des obstacles d'entrée proches, au-dessus desquels il doit passer pour se poser. Le passage de ces obstacles peut parfois être traumatisant pour l'élève, car il y a proximité de la queue de l'appareil avec ceux-ci, alors que la connaissance du gabarit de l'appareil n'est pas encore intériorisée. Cette utilisation du simulateur par le moniteur peut ainsi contribuer à la construction chez l'élève d'une nouvelle « image d'enveloppe corporelle » s'étendant au véhicule qu'il pilote.

\subsubsection{Articuler l'activité du formateur et celle de l'élève : les démonstrations}

Le couplage de l'activité de l'élève et celle du formateur peut être illustré en détail par le fonctionnement des démonstrations. Comme on l'a indiqué plus haut, l'activité attendue de l'élève lorsque le moniteur fait des démonstrations est « écouter / regarder /suivre aux commandes». Le moniteur commence le plus souvent les démonstrations sur simulateur, bien qu'il puisse montrer à nouveau en vol si l'élève a du mal à reproduire l'exercice. Dans les deux cas, la posture qu'il faut faire prendre à l'élève est de suivre comme si lui-même provoquait le mouvement des commandes qu'il perçoit et non se placer « en réaction » à l'action des commandes (c'est un problème qui peut être relevé dans des apprentissages de l'écriture où l'enfant résiste par réaction au geste de l'adulte qui veut le guider, et où l'aide supposée rate sa cible d'apprentissage).

Le moniteur sollicite d'abord l'élève auditivement: outre les sécurités qui doivent être annoncées systématiquement (comme en vol réel), l'élève entend le moniteur précommenter l'action avec un léger déphasage : schématiquement : il dit ce qu'il va faire et fait ce qu'il a dit, avec un très court délai entre parole et action. La cohésion entre situation sur simulateur et situation en vol réel tient fortement à la compétence de pilotage du moniteur et à sa compétence didactique (le timing du décalage dépend fortement des propriétés de la tâche de pilotage en jeu).

L'élève est ensuite sollicité visuellement : le moniteur explicite et fait apprendre le cycle de balayage visuel que l'élève doit adopter pour tel ou tel exercice. Ce balayage va de l'extérieur chercher des informations visuelles telles que, inclinaison, assiettes repères, etc., et à l'intérieur pour constater les modifications des éléments de vol (vitesse, altitude, cap...). Pour l'étayer, le moniteur montre avec le doigt, un peu comme un chef d'orchestre, les éléments que l'élève doit aller chercher : il réduit ainsi les degrés de liberté en orientant l'attention visuelle.

59 L'élève est enfin sollicité physiquement. Il perçoit trois choses avec les mains et les pieds (d'où l'importance des critères de coordination): l'ordre de l'action sur chacune des commandes (d'abord, puis simultanément, etc.), l'amplitude de cette action et la rapidité de chaque geste. C'est ce que nous désignerons du terme "modelage ", qui va au-delà de la « démonstration » dans l'analyse de l'étayage utilisée d'après Bruner. On espère qu'en règle générale cette posture de suivi de l'action ainsi modelée va se traduire par le fait que l'élève aura aussi mains et pieds « légers » lorsqu'il aura les commandes. En pratique l'élève peut être crispé aux commandes et « sur-contrôler » en faisant une action puis son contraire dans un cours laps de temps, ou une action trop ample suivie d'une action encore plus ample pour contrer l'action initiale, cela ayant pour effet d'amplifier les mouvements jusqu'à rendre l'appareil incontrôlé. 


\subsubsection{Utiliser le simulateur dans la formation à des tâches « délicates »}

60 Un rapport sur le programme de formation des pilotes de l'armée US signale que lors des premières heures de la formation au stationnaire les élèves continuent à surcontrôler l'hélico, perdent le contrôle et rendent les commandes à l'instructeur lorsqu'ils ont ainsi mis l'hélico dans une attitude dangereuse. La différence avec l'École réside dans le fait que l'élève ne rend pas les commandes: le moniteur l'aide si nécessaire en stabilisant la machine ou en limitant «en amont» le débattement des commandes. On retrouve une forme d'étayage de l'action où un composant du contrôle (ici moteur) est pris en charge par le moniteur. Cela jusqu'à ce que l'élève ait «le déclic» de la bonne tenue du stationnaire. On peut comparer cette action au moment où un enfant obtient pour la première fois l'équilibre sur son vélo. Bien entendu, de peu précis au début, mais maîtrisé, le stationnaire, au fur et à mesure du stage, devient un réflexe (un schème automatisé) et gagne en précision.

61 En fait, l'exercice demande un effort extrême à un élève : physiquement, car il est crispé, psychiquement, car cela demande beaucoup de ressources (prises d'informations extérieures fines), et psychologiquement, car la réussite à cet exercice en vol réel est un cap pour l'élève et le fait de ne pas arriver rapidement à passer ce cap l'angoisse. Le simulateur se montre un très bon outil pour dégrossir le travail ; il présente deux grands avantages : l'élève est moins stressé, car il sait qu'intrinsèquement il y a moins de danger, même si tout est fait pour rendre une séance immersive, et le simulateur est plus stable, car il ne subit pas d'aérologie (on travaille sans vent pour cet exercice en particulier). L'effet s'observe clairement: après huit séances sur simulateur, cumulant environ 40 minutes à travailler cette manœuvre, le stationnaire est quasiment acquis dès le premier vol réel, nonobstant la précision qui vient très rapidement, une fois le schème principal acquis, qui demande de " piloter dans une pièce de 2 euros ".

62 Mais là où en vol le stationnaire peut se travailler en milieu de séance pour rompre la routine du vol en cours, en simulation c'est la fin de séance qui doit être privilégiée : la proximité du sol appelle une corrélation entre visuel et postural qui ne peut pas avoir lieu dans le simulateur. On risque alors le mal du simulateur et la perte de l'élève (voire parfois du moniteur...) pour la séance ${ }^{20}$.

63 Le rôle du simulateur est assez similaire pour une autre manœuvre délicate propre à l'hélicoptère qu'est l'autorotation. L'outil présent à Dax n'est pas qualifié pour mener cette manœuvre à son terme, mais on peut y enseigner des composants essentiels du schème approprié : le réflexe de mise en autorotation, la descente et l'orientation de l'appareil jusqu'à une zone appropriée au poser, et le flare (amplitude du geste, hauteur sol), avec un apprentissage d'actes réflexes et de gestuelles «conformes». Le moniteur interrompt l'exercice à la fin du flare, car au-delà le modèle de vol ne respecte pas la réalité.

Le transfert vers le vol réel n'est toutefois pas dégagé de tout problème du fait du stress de l'élève qui peut générer des actions aux commandes inappropriées : flare trop haut ou trop bas, trop ample ou pas assez, etc. Du fait de la sensibilité à la variabilité situationnelle, chaque autorotation doit être effectuée dans un certain domaine de deux points de vue : d'un point de vue technique, pour assurer le bon déroulé de la finale en se situant dans une plage donnée des paramètres de l'action; d'un point de vue didactique, chaque moniteur ayant ses propres limites (réelles ou ressenties) de récupération et laissant donc plus ou moins d'initiative à son élève ; il s'entraîne d'ailleurs régulièrement 
en vol réel à la récupération de faute en autorotation. En conséquence, les incidents s'ils existent restent matériels, sans gravité et rares ${ }^{21}$.

Globalement, ce qui est fait au simulateur est fait en vol réel. Cependant, il y a des choses que l'on peut montrer au simulateur et qu'on ne peut pas faire en réel, à l'exemple de certaines pannes que l'on peut générer en simulation et que l'on ne peut produire en vol réel - à moins d'avoir effectivement cette panne. Certaines procédures de secours ou pannes sont jouées seulement au simulateur. En revanche, pour la panne moteur par exemple, la modélisation n'est pas conçue pour mener la panne jusqu'à son terme (poser au sol): le moniteur l'interrompt donc au moment du flare (fonction "gel»). Pour certaines pannes la modélisation n'est pas réaliste jusqu'au bout, donc on ne pratique pas certains exercices / pannes au risque d'injecter du negative training (constitution de schèmes inappropriés).

\subsection{6. Évaluer les élèves en continu selon des « facultés fondamentales »}

66 L'évaluation des élèves tout au long de la progression porte évidemment sur la conformité des schèmes au savoir de référence que constituent les procédures démontrées et à suivre. Elle porte aussi sur ce que Vidal-Gomel et Rogalski (2009) ont globalement catégorisé en " activité » et « comportement » des élèves en auto-école. La terminologie de l'ÉALAT est celle de "facultés fondamentales», qui couvrent un ensemble de dimensions. Dimensions perceptives liées au déplacement tridimensionnel de l'hélicoptère et aux propriétés du terrain et dimensions motrices: précision, ampleur et rythme (dosage), coordination. Dimensions attentionnelles, avec dissociation de l'attention sur la variation de plusieurs paramètres (flexibility dans les travaux anglophones). Dimensions décisionnelles, au sens large (Chauvin \& Rogalski, 2011) ; elles s'étendent du jugement de la situation (situation assessment) à la prise de décision stricto sensu avec choix d'une action et « engagement ferme dans la réalisation » (authoritatively execute a course of action, Adams \& Thompson, 1987). Le processus de traitement de la situation peut se situer à tous les niveaux du modèle de Rasmussen: réflexe du pilotage (niveau «skillbased» de Rasmussen), restitution de procédures connues bien choisies (niveau «rulebased »), résolution de problème avec recherche d'une solution adaptée au terrain, rapide et en sécurité (niveau « knowledge-based»), sans chercher l'optimalité.

Sont également évaluées des dimensions qu'on peut qualifier de "métacognitives », qui concernent les processus d'engagement et émotionnels, ainsi que la prise en compte de la sécurité propre (vol de l'hélico) et partagée (circulation), selon la lettre et l'esprit des procédures et règles. On peut y intégrer un autre composant "méta » : l'évaluation de ses propres compétences ${ }^{22}$. L'élève, après séance, remplit une fiche d'auto-analyse. Dans celle-ci, il va renseigner ses points forts ou positifs (ou tout du moins ce qui lui semble être comme tel), ses points faibles ou négatifs. De plus il y précise les éléments qui lui ont été conseillés par son moniteur pendant le vol. Le moniteur, quant à lui, va remplir une feuille de notation : après avoir évalué les différentes facultés fondamentales, il y écrit lui aussi les points forts (façon d'encourager); les points faibles sont décortiqués en trois points : «Faute, Origine, Remède ». La difficulté pour le moniteur réside dans l'action post-vol (ou post-simu) : théoriquement, à la fin du vol, lors du retour en salle de cours, le moniteur ne doit pas commencer par pré-débriefer l'élève. En effet, cette erreur pédagogique se solderait par une feuille d'auto-analyse élève conforme à ce que vient de lui dire son instructeur. La réussite du vol d'instruction sera complète si la feuille de note rédigée par le moniteur (il vaudrait plutôt l'appeler feuille de débriefing) concorde avec 
la feuille d'auto-analyse de l'élève. On pourra alors conclure que l'élève a «compris son vol », ses erreurs, comment les corriger, ainsi que la validation des acquis. Une fois ces documents remplis et comparés, le vrai débriefing peut commencer. Il permet de revenir sur certains points durs ou clés. L'évaluation s'effectue selon les mêmes grilles et appelle du moniteur les mêmes exigences en simulateur et en vol réel.

\section{Les apports pour la formation et pour la recherche} part, la conception du dispositif didactique a eu lieu très en amont de l'étude, qui ne visait pas à y contribuer. D'autre part, c'est la dimension technique (les «technical skills») d'une formation $a b$ initio qui a été centrale, au sens où la visée pour l'élève pilote est la maitrise de l'hélicoptère comme un instrument pour son activité professionnelle ultérieure ; nous n'avons pas considéré son intégration dans un dispositif opérationnel, impliquant la dimension collective de l'action ( «non technical skills», dans un sens très large, incluant la prise en compte du stress et des autres éléments intervenant dans les capacités opérationnelles des pilotes).

Le pilotage d'hélicoptère (dans le contexte de la formation étudiée) peut être situé dans le domaine de l'aviation et plus largement en tant que conduite d'un mobile, comme un cas où la dimension «sub-symbolique " de l'activité est essentielle : schématiquement, la dimension symbolique concerne un traitement conscient qui porte sur des représentations, mentales ou externes. Le niveau sub-symbolique intervient dans les processus fondamentaux de perception et d'action, dans la constitution des automatismes, dans les apprentissages sensori-moteurs et dans les apprentissages implicites. Le cas développé illustre l'interaction permanente entre ces niveaux dans la formation des compétences de base. À tout moment du processus de formation sont en effet visées et évaluées : des capacités perceptives liées au déplacement de l'hélicoptère et aux propriétés du terrain au-dessus duquel il évolue; des capacités motrices, en termes de coordination (actions simultanées mains-pieds sur des commandes), de précision et de dosage (ampleur et rythme) ; des capacités attentionnelles, en termes de distribution de l'attention (surveiller des informations externes et internes à l'hélicoptère par un balayage approprié) et de sensibilité aux variations des paramètres. Ces capacités qui impliquent la dimension sub-symbolique de l'activité interviennent dans la constitution des automatismes et des schèmes de pilotage. Une formule de Inhelder et Piaget (1966/2004) illustre bien la dimension sub-symbolique des schèmes : «la mémoire d'un schème est ce schème ». Elles s'intègrent par ailleurs dans des capacités décisionnelles (au sens large de la prise de décision), impliquant la dimension symbolique de l'activité.

71 L'activité du moniteur montre, d'une part, comment le simulateur lui sert d'instrument didactique pour faire apprendre le pilotage à l'élève et, d'autre part, comment fonctionne le processus d'étayage de l'activité de l'élève dans cette situation où les dimensions subsymbolique et symbolique sont en permanence intriquées. D'un point de vue méthodologique pour la recherche, l'étude montre qu'il faut prolonger les développements déjà proposés par Vidal-Gomel et Rogalski (2009) pour transposer le 
processus de tutorat de Bruner à l'étayage didactique pour couvrir tout type de contexte professionnel.

72 En revanche, le niveau métacognitif, réflexif, dont l'importance a été particulièrement soulignée par Pastré, n'est pas souligné (hors de la notion d'attitude) dans les guides spécifiant les tâches ou dans les actions du moniteur. Le fait que chaque séance soit autoévaluée par l'élève et que cette auto-évaluation soit mise en regard de celle du moniteur pourrait être plus développée du point de vue de l'activité du moniteur; on peut considérer que cette mise en regard prolonge la médiation, en la mettant en œuvre sur l'empan relativement long d'une séance. Par ailleurs, dans la formation à la conduite automobile, on a pu utiliser l'évolution des activités de guidage comme témoignant du développement des acquisitions des élèves (Boccara et al., 2015). On pourrait prolonger la question en s'interrogeant sur l'indicateur de développement que pourraient constituer les décalages possibles entre auto-évaluation et évaluation du formateur.

Par ailleurs, nous avons déjà souligné qu'on est en présence d'une situation de formation où un composant important de l'étayage est l'intervention directe du formateur sur l'activité sensori-motrice de l'élève. Cette intervention est rendue possible par le système «normal » de double poste de pilotage, en hélicoptère réel comme dans le simulateur. De ce point de vue, il n'y a donc pas de problème de transposition entre la situation visée de pilotage de l'hélicoptère réel et la situation de pilotage dans le simulateur. Dans d'autres situations de formation, la question pourrait se poser de concevoir une simulation avec simulateur introduisant une possibilité d'intervention didactique directe à travers des commandes en quelque sorte partagées. Une telle possibilité serait une forme de transposition didactique des situations cibles dans une autre dimension que celle qui est proposée par l'utilisation d'environnements virtuels de "réalité augmentée». Un problème commun qui se pose alors est celui de la gestion des effets de la transposition de la simulation au réel au niveau sub-symbolique de l'activité.

\section{Conclusion}

L'objet de ce chapitre a été l'étude d'un cas illustrant le rôle du formateur entre simulateur et "terrain», lors d'une formation initiale très technique, du fait des propriétés du pilotage d'hélicoptère, objet de l'apprentissage. La centration porte sur le premier des trois niveaux qui ont été définis dans des modèles issus de la recherche (Lefebvre, 2001, pour l'apprentissage de la conduite routière) ou de documents professionnels de formation (Adams \&Thompson, 1987, pour le pilotage d'hélicoptère). Le premier niveau est celui de la maîtrise instrumentale du mobile (le "pilotage" proprement dit); il concerne la manipulation des commandes, le contrôle et l'anticipation des lois physiques (inertie, trajectoires). Le second est celui de l'évolution dans un espace partagé, il concerne l'adaptation à l'environnement et l'identification des situations. Le dernier niveau technique est celui où la conduite du mobile vise à planifier puis réaliser un objectif à plus ou moins long terme (déplacement personnel ou professionnel, mission militaire ou civile). Ces différents niveaux sont emboîtés et interagissent fortement. Un quatrième composant est de nature métacognitive : il s'agit de la prise de conscience et des connaissances de son propre fonctionnement, en interaction avec le mobile piloté, l'environnement et la mission. 
une construction de schèmes de pilotage «robustes " que le futur pilote puisse activer dans les missions opérationnelles futures, malgré les perturbations qui marquent ces dernières ${ }^{23}$. Cela implique à côté des évaluations techniques, une évaluation formatrice orientée vers les dimensions motivationnelles et émotionnelles de l'activité des élèves, ainsi que leur prise en compte de la lettre et de l'esprit de la sécurité. L'étude s'est par ailleurs focalisée sur la dimension didactique de l'activité du formateur - ce qui ne signifie pas que la dimension pédagogique soit négligeable - et sans doute plus sensible aux caractéristiques personnelles des formateurs. En effet, chaque formateur, bien que formé à l'identique et cadré par l'intermédiaire d'une standardisation en tant que technicien du vol (démonstration de mécanismes, restitutions, synthèses), a toute latitude pour (et doit) adapter sa pédagogie à son élève (et aux conditions d'enseignement).

La prise en compte des conditions de l'activité du formateur a mis en évidence deux données. D'une part, elle dépend fortement de l'expertise propre du formateur dans le pilotage, du fait du rôle central des démonstrations étroitement suivies par les élèves, en préalable à leurs actions propres sur les commandes. Les connaissances fines du simulateur sont un second déterminant fort de la manière dont le moniteur fait passer l'élève du vol sur simulateur au vol réel : c'est une condition pour que le simulateur soit pour lui un instrument didactique, d'intervention sur l'élève, qu'il s'agisse de " dégrossissage ", d'amorce dans la constitution des schèmes de pilotage, d'apprentissage de traitement de situations non nominales - en évitant tout negative training. D'autre part, son activité se déploie avec un fort encadrement institutionnel, tenant, d'une part, à l'intégration du pilote dans l'organisation de l'aviation civile et, d'autre part, à la préparation d'un pilote d'hélicoptère militaire devant s'insérer dans des missions opérationnelles. Cet encadrement se traduit par un guidage de l'activité didactique du formateur, qui constitue un système à la fois de contraintes et de ressources.

L'analyse de ces guides, comme celle de l'activité du formateur, a mis en évidence une orientation de la formation qu'on peut situer dans la lignée de Vygotsky, où l'apprentissage appelle la médiation du formateur mieux sachant, qui va étayer l'activité propre de l'élève selon un processus analysé par Bruner pour les apprentissages chez l'enfant et transposable pour la formation professionnelle. L'apport du cas étudié à la recherche tient en particulier à la prise en compte dans cet étayage de toutes les dimensions cognitives de l'activité de l'élève: perceptivo-motrices, attentionnelles et décisionnelles au sens large (du jugement sur la situation en cours à une décision d'action cohérente et déterminée). Un prolongement dans l'étude devrait concerner la dimension pédagogique, pour spécifier les composants de l'étayage touchant à l'enrôlement de l'élève, le maintien de son engagement avec la gestion de la frustration et du stress.

Enfin, des études seraient évidemment utiles pour identifier les invariants ou les différenciations dans le rôle des formateurs entre simulation et réel, selon qu'on peut se centrer sur l'activité didactique sur simulateur vs terrain ou si au-delà du niveau technique de l'activité-cible, on s'intéresse à la gestion par les formateurs au long de la formation des relations entre situations de simulation et missions professionnelles. 


\section{BIBLIOGRAPHIE}

Adams, R., \& Thompson, J. (1987). Aeronautical decision making for helicopter pilots. Training Manual. National Technical Information Service. Springfield, Virginia 22161.

Bellenkens, A.H., Wickens, C.D., \& Kramer, A.F. (1997). Visual scanning and pilot expertise: the role of attentional flexibility and mental model development. Aviation, Space, and Environmental Medicine, 68(7), 569-579.

Boccara, V., Vidal-Gomel, C., Rogalski, J., \& Delhomme, P. (2015). A longitudinal study of student's driving skills development from trainers' guidance. Applied Ergonomics, 46, 21-29.

Bruner, J. (2002). Le développement. Savoir faire. Savoir dire. Paris : Presses Universitaires de France.

Chauvin, C., \& Rogalski, J. (2011). Les modèles de la macro cognition : intérêts et limites. Colloque du GDR CNRS Psychologie Ergonomique, Toulouse, 4-7 avril 2011.

Collet, C. (2002). Mouvement \& Cerveau. Neurophysiologie des activités physiques et sportives. Paris : DeBoeck Université.

DGAC (2001). Guide Facteurs Humains pour l'instructeur. Mérignac : Service de l'information aéronautique.

Grau, Y., Doireau, P., \& Poisson, R. (1998). Conception et utilisation de la simulation pour la formation : pratiques actuelles dans le domaine militaire. Le Travail Humain, 61(4), 361-385.

Guillot, A., \& Collet, C. (2013). Imagerie motrice : principes, concepts et méthodes. Éditorial. Movement \& Sport Sciences - Science \& Motricité, 82, 1-6.

Harris, F.D., Kasper, E.F., \& Iseler, L.E. (2000). U.S. civil rotorcraft accidents, 1963 through 1997. NASA/TM-2000-209597. USAAMCOM-TR-00-A-006. Ames Research Center. Moffett Field, CA.

Hays, R. T., Jacobs, J. W., Prince, C., \& Salas, E. (1992). Flight simulator training effectiveness: A meta-analysis. Military Psychology, 4(2), 1992, 63-74.

Inhelder, B., \& Piaget, J. (1966/2004). La psychologie de l'enfant. Paris : PUF.

Jeannerod, M. (2009). Le cerveau volontaire. Paris : Odile Jacob.

Lefebvre, C. (2001). Vers une formation à la conduite automobile intégrant des connaissances conceptuelles et métaconnaissances. Recherche Transports Sécurité, 70, 16-40.

Lépinard, P. (2014). Du serious au full flight simulator : proposition d'un cadre conceptuel commun pour la formation des formateurs en simulation. Systèmes d'information \& management, 19(3), 39-68.

Leplat, J. (1997). Regards sur l'activitgamingé en situation de travail. Paris : PUF.

McCauley, M. (2006). Do army helicopter training simulators need motion bases? Technical Report 1176. Arlington, VI: US Army research institute for the behavioral and social sciences.

Padfield, R. (1992). How to fly helicopters (2 édition 2013). McGraw Hill Professional.

Pastré, P. (Ed.) (2005). Apprendre par la simulation: de l'analyse du travail aux apprentissages professionnels. Toulouse : Octarès.

Pastré, P. (2011). La didactique professionnelle. Approche anthropologique du développement chez l'adulte . Paris : PUF. 
Pastré, P., Mayen, P., \& Vergnaud, G. (2006). La didactique professionnelle. Revue française de Pédagogie, 154, 145-198.

Proctor, M. D., Bauer, M., \& Lucario, T. (2007). Helicopter flight training through serious aviation gaming. The Journal of Defense Modeling and Simulation, 4, 405-425.

Rizzolatti, G., \& Sinigaglia, C. (2006). Les neurones miroirs. Paris : Odile Jacob.

Robinski, M., \& Stein, M. (2013). Tracking visual scanning techniques in training simulation for helicopter landing. Journal of Eye Movement Research, 6(2), 3, 1-17.

Rogalski, J. (2003). Y a-t-il un pilote dans la classe ? Une analyse de l'activité l'enseignant comme gestion d'un environnement dynamique ouvert. Recherches en didactique des mathématiques, 23 , 343-388.

Rogalski, J. (2011). Expériences et construction d'invariants : connaissances opérationnelles, schèmes d'action et « qualités ». Travail et Apprentissages, 7, 45-61.

Salas, E., Bowers, C.A., \& Rhodenizer, L. (1998). It is not how much you have but how you use it: Toward a rational use of simulation to support aviation training. The International Journal of Aviation Psychology, 8(3), 197-208.

Samurçay, R., \& Rabardel, P. (2004). Modèles pour l'analyse de l'activité, des compétences. Propostions. In R. Samurçay \& P. Pastré (Eds.), Recherches en didactique professionnelle (pp. 163-187). Toulouse : Octarès.

Samurçay, R., \& Rogalski, J. (1998). Exploitation didactique des situations de simulation. Le Travail Humain, 61(4), 333-359.

Savoyant, A. (2005). L'activité en situation de simulation : objet d'analyse et moyen de développement. In P. Pastré (Ed.), Apprendre par la simulation (pp. 41-54). Toulouse : Octarès.

Stewart II, J.A., Dohme, J.A., \& Nullmeyer, R.T. (2002). U.S. army initial entry rotary-wing transfer of training research. The International Journal of Aviation Psychology, 12(4), 359-375.

Sullivan, J, Yang, J.H., Day, M., \& Kennedy, Q. (2011). Training simulation for helicopter navigation by characterizing visual scan patterns. Aviation, Space, and Environmental Medicine, 82, 871-878.

Transport Canada (2006). Manuel de pilotage des hélicoptères. Canada : Ministère des transports.

Valot, C., Grau, J.Y., \& Amalberti, R. (1993). Les métaconnaissances : des représentations de ses propres compétences. In A. Weill-Fassina, P. Rabardel, \& D. Dubois (Eds.), Représentations pour l'action (pp. 271-293). Toulouse : Octarès.

Vidal-Gomel, C., Boccara, V., Rogalski, J., \& Delhomme, P. (2008). Les activités de guidage des formateurs au cours d'un audit destiné à des conducteurs expérimentés et âgés. Travail et Apprentissages, 2, 46-64.

Vidal-Gomel, C., \& Rogalski, J. (2009). Analyser l'activité des formateurs en conduite automobile. Une étude exploratoire des aspects collectifs du travail. Savoirs, 20, 85-118.

Vygotski, L. (1997). Pensée et Langage. Paris : La dispute.

Wood, D., Bruner, J. S., \& Ross, G. (1976). The role of tutoring in problem solving. Journal of Child Psychology and Psychiatry, and Allied Disciplines, 17(2), 89-100.

Yang, J.H., Kennedy, Q., Sullivan, J., \& Fricker, R.D. (2013). Pilot performance: Assessing how scan patterns \& navigational assessments vary with flight expertise. Aviation, Space, and Environmental Medicine, 84(2), 116-124. 


\section{NOTES}

1. Analogue à celle de Commandant de bord et officier dans le cadre de l'aviation civile.

2. Le terme de formateur est utilisé à un niveau générique. Il vise tout intervenant dans une formation professionnelle, ici celle de pilote d'hélicoptère militaire. Le terme de moniteur est employé par l'institution pour les intervenants spécifiquement chargés de la formation aux manœuvres de pilotage utilisant le simulateur, dans une relation "un-à-un » avec les élèvespilotes.

3. Les futurs pilotes (en général, internes) sont par ailleurs formés à des dimensions techniques du fonctionnement des hélicoptères et de leur pilotage, ainsi qu'à la dimension militaire de leur futur engagement, et à d'autres domaines généralistes.

4. Dans un rapport pour l'armée US, McCauley (2006) rappelle les nombreux travaux montrant les transferts positifs à partir de la formation sur simulateur (pour les avions, mais peu d'études pour les hélicoptères), et relève qu'on trouve peu de données montrant l'importance de l'intégration du mouvement dans le simulateur.

5. L'étude a été engagée dans le contexte d'un groupe de travail sur la simulation dans la formation, SimAFor, faisant interagir des formateurs, des chercheurs et des concepteurs.

6. «Learning to fly helicopters is generally considered to be the most challenging and difficult aviation task ».

7. Il s'agit des trois axes des mouvements propres de l'hélicoptère - tangage, roulis, cadence plus l'axe vertical (en jeu lorsque la puissance n'est pas automatiquement régulée).

8. « unlike pilots of their fixed-wing counterparts, the helicopter pilot must constantly maintain control of four primary axes that are frequently tightly coupled [...] A pilot must, therefore, use both feet and both hands"

9. «more recent research in this area has been the exception, not the rule. This is especially true for rotary-wing aircrew training research »

10. Il est à noter que les simulateurs EFI de Dax sont très réalistes du point de vue de la fidélité des commandes; la qualité de la base de données visuelles pourrait, pour un visiteur extérieur, manquer de réalisme : l'EFI ayant été développé pour l'apprentissage de mécanismes, le rendu des paysages est accessoire. En revanche, les implantations, les dimensions des objets, etc. concordent avec le réel.

11. " as way of epilogue, Bristow Academy has since adopted a Bell 206 flight simulator as part of their training program and none of the three generic-game approaches considered above »

12. Une proposition de s'appuyer sur des outils d'eye tracking (Robinski \& Stein, 2013) pose des questions d'opérationnalité - et semble davantage orientée vers la recherche que la formation, quoiqu'en dise le titre de l'article.

13. Le terme retenu à l'EALAT est « dissociation de l'attention ». Le balayage est un moyen pour récupérer des informations (paramètres / position de l'appareil). Il est censé améliorer la dissociation de l'attention qui, elle, évalue la capacité de l'élève à observer plusieurs données et en déduire les actions appropriées. Anticipant sur la suite, nous devons préciser ici qu'à l'école de Dax les moniteurs apprennent aux élèves les balayages visuels - ils diffèrent en fonction des exercices et/ou du type de vol. Pour confirmer l'existence de leur balayage, outre le résultat des actions (résultat), le moniteur s'attache à regarder son élève (et plus précisément son regard). C'est un élément très important pour détecter une faille dans l'apprentissage de certains réflexes. 14. Les trois autres concernent l'engagement et le maintien dans la tâche - ce qui renvoie à l'enrôlement (cf. plus haut), et le contrôle de la frustration (liée au non-succès).

15. En particulier il faut tenir compte des limites dans la modélisation du vecteur simulé : par exemple, des différences notoires existent (cas de l'EFI de Dax) dans la finale d'autorotation où 
l'atterrissage post flare, (manœuvre consistant à diminuer la vitesse horizontale en cabrant l'appareil), n'est pas conforme au réel. Le moniteur "gèle » donc la simulation après le flare. Poursuivre au-delà générerait du negative training.

16. Manœuvre est utilisé ici comme un terme générique, pouvant couvrir des procédures plus ou moins élémentaires mais aussi des tâches complexes. Le terme exercice renvoie davantage à la dimension formation qu'à celle pilotage.

17. De nombreuses recherches initiées par l'identification de l'existence de neurones miroirs et de neurones prémoteurs (Rizzolatti \& Sinigaglia, 2006) ont mis en évidence cette construction d'une véritable simulation motrice qui peut être exploitée pour les apprentissages (Collet, 2002 ; Guillot \& Collet, 2013 ; Jeannerod, 2009).

18. Le rollover est une réaction de l'appareil qui lorsqu'il est ancré au sol - par un patin ou une roue - pivote autour de cet axe indésirable. Lorsque le centre de gravité bascule au-delà d'une certaine position, l'appareil ne peut plus être arrêté dans sa rotation et crashe.

19. " before performing operations in a mud/ muskeg / toundra environment, it is important to understand dynamic rollover characteristics"

20. l'élève ayant le mal du simulateur (nausées ou plus, etc.) est incapable de continuer à piloter (si cela arrive au moniteur, bien que plus aguerri, il ne peut plus intervenir pour la formation...)

21. En gros, sur 100000 heures de vol, 3 casses importantes et quelques « béquilles » tordues.

22. Adams et Thompson (1987) soulignaient déjà dans leur manuel de formation : « how a pilot handles responsibilities [...] also depends to a large degree upon attitudes - toward safety, toward oneself, and toward flying [...] Attitudes are learned and good attitudes can be developed, through training, into a positive mental framework that encourages and produces good pilot decision making. "

23. Parmi les interventions "méta", l'auteur formateur explique aux élèves que ce qu'ils apprennent à l'École, s'ils le valident durablement en se forçant toujours à effectuer une approche, un décollage ou tout autre exercice de manière correcte, sera ancré en eux et qu'ils pourront en cas de « surchauffe » se raccrocher à ces « basiques".

\section{RÉSUMÉS}

L'étude interroge l'activité de formation sur simulateur pour l'acquisition des compétences cibles en situation réelle. Elle s'appuie sur le cas de la formation initiale au pilotage d'hélicoptères militaires. Cette formation très encadrée articule séances sur simulateur et vols réels, visant à la maîtrise des manœuvres au cœur du pilotage. L'activité du formateur est également très encadrée avec une organisation préalable des tâches, un guidage pour le déroulement des séances, évaluation comprise. La référence théorique retenue est celle de la didactique professionnelle (Pastré, Mayen, \& Vergnaud, 2006). La médiation didactique exercée par le formateur est analysée en transposant la notion d'étayage théorisée par Bruner (2002). Les données de la littérature sur la conduite de mobiles sont convoquées pour situer les questions ouvertes sur la place de la simulation dans la formation. Le rôle des moniteurs dans l'articulation des compétences des élèves sur simulateur et sur le terrain est traité dans une étude de cas qui s'appuie sur l'expertise du second auteur (pilote, formateur et concepteur d'outils pour les moniteurs). On vise en particulier à préciser l'activité d'étayage selon les situations. La discussion porte sur le domaine de pertinence des analyses proposées pour les apprentissages dans des types de situations professionnelles marquées par la place des activités sensori-motrices, par la 
complexité des interactions entre commandes d'action, et par les relations entre procéduralisation des tâches et ouverture de l'environnement dynamique.

The paper aims at highlighting the links between simulator training and direct training in the case of initial training for military helicopter pilots. The issue is considered from the perspective of the instructor's activity. Pilot students are severely selected and their training is strongly framed, both through the civil aviation norms and by the high expectations vis-à-vis military pilots. It includes simulation sessions and real flights, in order that students master the maneuvers which are the core of using helicopter as an instrument, a means among others for military missions. Instructors intervene in both types of training. Their task is itself strictly framed: organization of training tasks, precise guidelines for exercises and sessions management, including evaluation. Professional Didactics (Pastré, Mayen, \& Vergnaud, 2006) provides a theoretical framework for studying the role of instructors in coordinating students' training. The notion of scaffolding - transposed from Bruner's notion of tutoring (Wood, Bruner, \& Ross, 1976) - offers an entry on their didactical mediation. Professional documents and research productions in the aviation or driving domains are used for identifying actual knowledge and open questions about training. Their analyses orient the study developed in the last part of the paper about the role played by instructors in relating students' competences acquired in the simulator and on the field. The personal expertise of the second author - as a pilot, a trainer and a monitors' trainer is deeply involved for tackling this issue. The conclusion discusses the relevance domain for such an analysis of trainers' activity, when work implies sensori-motor competences, involves intrinsic complexity of the interactions between commands, where tasks are highly proceduralised but situations openness calls for developing anticipation competences.

\section{INDEX}

Keywords : aviation, dynamic environment management, training, simulation, trainer's activity Mots-clés : aviation, gestion d'environnement dynamique, formation, simulation, activité du formateur

\section{AUTEURS}

\section{JANINE ROGALSKI}

Laboratoire de Didactique André Revuz (LDAR EA 4434) UFR de mathématiques - Université Paris Diderot Bâtiment Sophie Germain - $8^{\mathrm{e}}$ étage Place Aurélie Nemours 75013 Paris rogalski.muret@gmail.com

\section{BORIS COLIN}

Base école Général Navelet, Route de Tercis, 40180 Dax - boris.colin@intradef.gouv.fr 